\title{
A SYSTEM OF QUADRICS DESCRIBING THE ORBIT OF THE HIGHEST WEIGHT VECTOR
}

\author{
WOODY LICHTENSTEIN
}

\begin{abstract}
Let $G$ be a complex semisimple Lie group acting irreducibly on a finite dimensional vector space $V$. A simple method is given for constructing a system of quadratic equations which defines the orbit of the highest weight vector in the projective space $P V$.
\end{abstract}

1. The Borel-Weil Theorem gives a method for constructing all irreducible representations of a compact connected semisimple Lie group $G$. Any such representation arises as the action of $G$ on the space of homogeneous polynomials of fixed degree restricted to a $G$-homogeneous projective variety. (Of course, since it does not really make sense to restrict a homogeneous polynomial to a projective variety $\mathcal{V}$, the vector space in question is usually described as the space of holomorphic sections of a holomorphic line bundle over $\mathcal{T}$.) The results of Wang [11], Lichnerowicz [5] and Borel and Weil [8] show that any irreducible projective variety $\mathcal{V}$ with a finite fundamental group and a transitive group action must be of the form $\mathfrak{V}=G_{\mathbf{C}} / P_{\mathbf{C}}$ where $G_{\mathbf{C}}$ is a connected complex semisimple Lie group (the complexification of the compact group $G$ ) and $P_{\mathrm{C}}$ is a parabolic subgroup (a subgroup containing a maximal connected solvable subgroup). Furthermore every space $G_{\mathrm{C}} / P_{\mathrm{C}}$ can be described as the orbit of the line spanned by the highest weight vector in a finite dimensional irreducible representation of $G_{\mathbf{C}}$.

All the varieties $G_{\mathrm{C}} / P_{\mathrm{C}}$ may be easily constructed once one understands those for which $P_{\mathrm{C}}$ is a maximal subgroup. (These correspond to the orbits of the highest weight vectors in fundamental representations.) For example, when $G_{C}=\operatorname{SL}(n, C)$ the fundamental representations occur on the spaces $\wedge^{k}\left(\mathbf{C}^{n}\right), k=1, \ldots, n-1$, where $G_{\mathbf{C}}$ acts naturally on $\mathbf{C}^{n}$. The orbit of the highest weight vector in $P \wedge^{k}\left(\mathbf{C}^{n}\right)$ - the corresponding projective space-is the Grassmann manifold $G_{k, n}$ of $k$-planes in $n$-space. It is well known that the variety $G_{k, n} \subset P \wedge^{k}\left(\mathbf{C}^{n}\right)$ is cut out by a system of quadrics, the Plücker equations [3]. With this as a starting point it is not hard to show that for every fundamental representation of a classical group the orbit of the highest weight vector is cut out by a system of quadrics.

For the exceptional groups it is more difficult to determine the corresponding varieties so explicitly. Nevertheless, in many cases it is known that these varieties are cut out by quadrics. In particular, the orbit of the highest weight vector in the 27

Received by the editors February 11, 1981 and, in revised form, August 24, 1981; presented at the NSF-CBMS Regional Conference on Representations of Semisimple Lie Groups, Chapel Hill, North Carolina, May 1981.

1980 Mathematics Subject Classification. Primary 22E10, 32M10. 
dimensional representation of $E_{6}$ can be identified with the Cayley projective plane $[1,9,10]$ which in turn can be identified with the rank 1 elements in the Jordan algebra of hermitian $3 \times 3$ Cayley matrices [4]. The condition imposed by rank 1 is that all $2 \times 2$ minors vanish. This is a system of quadrics. Freudenthal has also given a system of quadrics defining the orbit of the highest weight vector in the 56 dimensional representation of $E_{7}$ [2].

In this note a simple method is given for constructing a system of quadrics defining the orbit of the highest weight vector in any irreducible representation.

I would like to acknowledge that the conjecture that every $G_{\mathrm{C}} / P_{\mathrm{C}}$ is cut out by quadrics was arrived at in conversations with Harsh Pittie.

2. Let $G$ be a connected complex semisimple Lie group of rank $l$ with Lie algebra $\mathfrak{g}$ and universal enveloping algebra $U(\mathrm{~g})$. Let $V_{\lambda}$ be a $\mathrm{C}$-vector space on which $G$ acts via the finite dimensional irreducible representation with highest weight $\lambda$. Let $\mathfrak{h} \subset \mathfrak{g}$ be a Cartan subalgebra, let $\left\{X_{\alpha}\right\}$ be the set of positive root vectors (for some choice of ordering of the roots) and let $\left\{X_{-\alpha}\right\}$ be the negative root vectors, chosen so that $B\left(X_{\alpha}, X_{-\alpha}\right)=1$ where $B$ is the Killing form. Let $H_{i}, i=1, \ldots, l$, be a $B$-orthonormal basis of $\mathfrak{h}$. Set

$$
\Omega=\sum_{\alpha>0}\left(X_{\alpha} X_{-\alpha}+X_{-\alpha} X_{\alpha}\right)+\sum_{i=1}^{l} H_{i}^{2}
$$

the Casimir operator in $U(\mathfrak{g})$. In fact $\Omega$ is in the center of the enveloping algebra and therefore acts on any irreducible representation as multiplication by a scalar. Specifically, by checking on the highest weight vector, one can see easily that on $V_{\lambda}$, $\Omega$ acts as multiplication by $\langle\lambda+\delta, \lambda+\delta\rangle-\langle\delta, \delta\rangle=\langle\lambda+2 \delta, \lambda\rangle$ where $\delta$ is half the sum of the positive roots and $\langle\cdot, \cdot\rangle$ is the Killing form translated to the dual space $\mathfrak{h}^{*}$ of $\mathfrak{h}$.

THEOREM. The system of quadrics

$$
\Omega(v \otimes v)=\langle 2 \lambda+2 \delta, 2 \lambda\rangle(v \otimes v)
$$

characterizes the orbit of the highest weight vector.

[Note that both sides of (1) are elements of $V_{\lambda} \odot V_{\lambda}$ (symmetric tensor square of $V_{\lambda}$ ) and thus if $d_{\lambda}=\operatorname{dim} V_{\lambda}$, (1) may be thought of as a system of $\left(d_{\lambda}+1\right) d_{\lambda} / 2$ quadratic equations in the $d_{\lambda}$ components of $v$.]

Proof. The system (1) certainly holds when $v$ is the highest weight vector $v_{\lambda}$ since then $v_{\lambda} \otimes v_{\lambda}$ is in a subspace of $V_{\lambda} \otimes V_{\lambda}$ which transforms under $G$ according to the irreducible representation with highest weight $2 \lambda$. But then (1) must hold everywhere on the $G$-orbit $\mathcal{O}_{v_{\lambda}}$ of $v_{\lambda}$. Let $\pi: V_{\lambda}-\{0\} \rightarrow P V_{\lambda}$ be the canonical map to projective space. Since $G$ acts transitively on the variety $\mathscr{V}=\pi\left(\theta_{v_{\lambda}}\right), \mathcal{V}$ is nonsingular and contained in $\pi(\mho)$ where $W$ is the algebraic set defined by (1). By calculation of the tangent space of $\mathscr{V}$ at $\pi\left(v_{\lambda}\right)$ it can be shown that $\operatorname{dim} \mathcal{V}=$ \# positive roots $\alpha$ such that $\langle\lambda, \alpha\rangle \neq 0,=n_{\lambda}$. If we rewrite (1) in the form $F(v)=0$ where $F: V_{\lambda} \rightarrow V_{\lambda}\left(V_{\lambda}\right.$ then we can show $V=\pi(W)$ if we can show that Rank $D F\left(v_{\lambda}\right) \geqslant d_{\lambda}-1-n_{\lambda}$. The reason this suffices is as follows. 
First, $\mathscr{W}$ is a union of $G$-orbits, and, if $x \in V_{\lambda}$ is any vector with $G$-orbit $\theta_{x}$, then $\pi\left(\theta_{x}\right)$ contains $\pi\left(v_{\lambda}\right)$ in its closure. [Since $V_{\lambda}$ is irreducible, every orbit $\theta_{x}$ spans $V_{\lambda}$, so we may assume $x=c v_{\lambda}+y$ with $c \neq 0$ and $y$ a linear combination of weight vectors of weight less than $\lambda$. Now choose $H \in \mathfrak{h}$ such that $\lambda(H)>0$ and $\alpha(H)>0$ for all positive roots $\alpha$. Then $\operatorname{Lim}_{t \rightarrow \infty} e^{-t \lambda(H)}\left(e^{t H} \cdot x\right)=c v_{\lambda}$.]

Now either Rank $D F$ is constant on some neighborhood of $v_{\lambda}$ in $\mathscr{W}$, or there are points arbitrarily near $v_{\lambda}$ in $थ$ where $\operatorname{Rank} D F>\operatorname{Rank} D F\left(v_{\lambda}\right) \geqslant d_{\lambda}-1-n_{\lambda}$. The second case is impossible since any orbit of a point near $v_{\lambda}$ will have dimension $\geqslant n_{\lambda}+1$. In the first case we may apply the implicit function theorem to conclude that there is a neighborhood $\mathcal{Q}$ of $\pi\left(v_{\lambda}\right)$ such that $\pi(\mathscr{\mho}) \cap \mathcal{Q}$ is a smooth connected $n_{\lambda}$-dimensional manifold containing the smooth $n_{\lambda}$-dimensional manifold $\mathcal{V} \cap \mathcal{Q}$. It follows that the component of $\pi(\mathcal{W})$ through $\pi\left(v_{\lambda}\right)$ is $\mathcal{V}$. But the above argument concerning orbits in $P V_{\lambda}$ shows that any other component of $\pi(\mathcal{W})$ would have to intersect $\mathscr{U}$, which is impossible, so that $\pi(\mathscr{W})$ is irreducible and $\pi(\mathscr{W})=\mathscr{V}$.

We proceed to rewrite (1) as

$$
\begin{aligned}
\Omega v \otimes v+v \otimes \Omega v & +2 \sum_{\alpha>0}\left(X_{\alpha} v \otimes X_{-\alpha} v+X_{-\alpha} v \otimes X_{\alpha} v\right) \\
& +2 \sum_{i=1}^{l} H_{i} v \otimes H_{i} v-\langle 2 \lambda+2 \delta, 2 \lambda\rangle(v \otimes v)=0 .
\end{aligned}
$$

Using $\Omega v=\langle\lambda+2 \delta, \lambda\rangle v$ this simplifies to

$$
\begin{aligned}
-\langle\lambda, \lambda\rangle(v \otimes v)+\sum_{\alpha>0}\left(X_{\alpha} v \otimes X_{-\alpha} v+X_{-\alpha} v \otimes X_{\alpha} v\right) \\
+\sum_{i=1}^{l} H_{i} v \otimes H_{i} v=F(v)=0 .
\end{aligned}
$$

Finally, we calculate $D F\left(v_{\lambda}\right): V_{\lambda} \rightarrow V_{\lambda} \odot V_{\lambda}$.

$$
\begin{aligned}
\left.\frac{d}{d t}\right|_{t=0} F\left(v_{\lambda}+t x\right)=-\langle\lambda, \lambda\rangle\left(v_{\lambda} \otimes w+w \otimes v_{\lambda}\right) \\
+\sum_{\alpha>0}\left(X_{\alpha} w \otimes X_{-\alpha} v_{\lambda}+X_{-\alpha} v_{\lambda} \otimes X_{\alpha} w\right) \\
+\sum_{i=1}^{l}\left(H_{i} v_{\lambda} \otimes H_{i} w+H_{i} w \otimes H_{i} v_{\lambda}\right) .
\end{aligned}
$$

Take $w \in V_{\lambda}$ to be a weight vector of weight $\mu$. Then

$$
\begin{aligned}
D F\left(v_{\lambda}\right)(w)= & \langle\lambda, \mu-\lambda\rangle\left(v_{\lambda} \otimes w+w \otimes v_{\lambda}\right) \\
& +\sum_{\alpha>0}\left(X_{\alpha} w \otimes X_{-\alpha} v_{\lambda}+X_{-\alpha} v_{\lambda} \otimes X_{\alpha} w\right)
\end{aligned}
$$

The right-hand side of (3) is a weight vector in $V_{\lambda}$ (5) $V_{\lambda}$ of weight $\lambda+\mu$. The coefficient of $v_{\lambda} \otimes w+w \otimes v_{\lambda}$ is nonzero for $\mu \neq \lambda$. If the weight $\mu$ is not of the form $\lambda-\alpha$ for any positive root $\alpha$, then the sum term on the right-hand side of (3) is linearly independent from $v_{\lambda} \otimes w+w \otimes v_{\lambda}$. If $\mu$ is of the form $\lambda-\alpha$ and $w$ is 
orthogonal to $X_{-\alpha} v_{\lambda}$ then the same statement holds. Since $n_{\lambda}$ is the number of weights of $V_{\lambda}$ of the form $\lambda-\alpha$, it is now easy to see that there are at least $d_{\lambda}-1-n_{\lambda}$ linearly independent vectors in the image of $D F\left(v_{\lambda}\right)$.

\section{REFERENCES}

1. D. Drucker, Exceptional Lie algebras and the structure of Hermitian symmetric spaces, Mem. Amer. Math. Soc. No. 208, 1978.

2. H. Freudenthal, Sur le groupe exceptionnel $E_{7}$, Nederl. Akad. Wetensch. Proc. Ser. A 56 (1953), 81-89.

3. P. Griffiths and J. Harris, Principles of algebraic geometry, Wiley, New York, 1978.

4. N. Jacobson, Structure and representations of Jordan algebras, Amer. Math. Soc., Providence, R. I., 1968.

5. A. Lichnerowicz, Sur les espaces homogènes Kählériens, C. R. Acad. Sci. Paris 237 (1953), 695-697.

6. R. Marlin, Anneaux de Chow des groupes algébriques $\operatorname{Su}(n), \operatorname{Sp}(n), \operatorname{SO}(n), \operatorname{Spin}(n), G_{2}, F_{4}, \mathrm{C} . \mathrm{R}$. Acad. Sci. Paris 279 (1974), 119-122.

7. D. Montgomery, Simply connected homogeneous spaces, Proc. Amer. Math. Soc. 1 (1950), 467-469.

8. J. P. Serre, Représentations linéaires et espaces homogènes Kählériens des groupes de Lie compacts, Séminaire Bourbaki No. 100, 1954.

9. J. Tits, Le plan projectif des octaves et les groupes exceptionnels $E_{6}$ et $E_{7}$, Acad. Roy. Belg. Bull. $\mathrm{Cl}$. Sci. 40 (1954), 29-40.

10. Sur certaines classes d'espaces homogènes de groupes de Lie, Acad. Roy. Belg. Cl. Sci. Mem. Collect. 29 (1955-56).

11. H. C. Wang, Closed manifolds with homogeneous complex structure, Amer. J. Math. 76 (1954), 1-32.

Department of Mathematics, University of Georgia, Athens, Georgia 30602 